\title{
Hybrid Reliability Analysis for Spacecraft Docking Lock with Random and Interval Uncertainty
}

\author{
Jianguo Zhang, ${ }^{1,2}$ Jiwei Qiu,, ${ }^{1,2}$ and Pidong Wang ${ }^{3}$ \\ ${ }^{1}$ School of Reliability and Systems Engineering, Beihang University, Beijing 100191, China \\ ${ }^{2}$ Science and Technology on Reliability and Engineering Laboratory, Beihang University, Beijing 100191, China \\ ${ }^{3}$ Department of Industrial Engineering, Tsinghua University, Beijing 100084, China \\ Correspondence should be addressed to Pidong Wang; wpd-pizi@163.com
}

Received 4 July 2017; Accepted 2 October 2017; Published 31 October 2017

Academic Editor: Christopher J. Damaren

Copyright (c) 2017 Jianguo Zhang et al. This is an open access article distributed under the Creative Commons Attribution License, which permits unrestricted use, distribution, and reproduction in any medium, provided the original work is properly cited.

\begin{abstract}
This paper presents a novel procedure based on first-order reliability method (FORM) for structural reliability analysis with hybrid variables, that is, random and interval variables. This method can significantly improve the computational efficiency for the abovementioned hybrid reliability analysis (HRA), while generally providing sufficient precision. In the proposed procedure, the hybrid problem is reduced to standard reliability problem with the polar coordinates, where an $n$-dimensional limit-state function is defined only in terms of two random variables. Firstly, the linear Taylor series is used to approximate the limit-state function around the design point. Subsequently, with the approximation of the $n$-dimensional limit-state function, the new bidimensional limit state is established by the polar coordinate transformation. And the probability density functions (PDFs) of the two variables can be obtained by the PDFs of random variables and bounds of interval variables. Then, the interval of failure probability is efficiently calculated by the integral method. At last, one simple problem with explicit expressions and one engineering application of spacecraft docking lock are employed to demonstrate the effectiveness of the proposed methods.
\end{abstract}

\section{Introduction}

In recent years, reliability analysis has been a research hotspot of structural design and analysis. The influence of uncertainty arising in loads, material properties, dimensions, and geometry becomes more and more profound [1]. Probability theory is one of the most universal tools to estimate structural reliability and safety by calculating the probability of failure $P_{\mathrm{f}}$ and the reliability index $\beta[2-4]$. Hence, FORM is the traditional and most commonly used method in the field of structural reliability, and it has been widely employed in a wide range of industrial fields [5-8].

In FORM for structural reliability analysis, by the firstorder Taylor series, the limit-state function is approximated at the design point in the standard Gauss space of transformed independent random variables $[9,10]$. In this space, reliability analysis is usually converted into an optimization problem to solve the reliability index [8]. A review of FORM can be found in Breitung [11]. Its application and accuracy in most structure engineering are well recognized, for instance by Rackwitz [12]. Derived from FORM, many probability approximation methods have further developments and more refined alternatives [11-15]. However, some problems may arise in the application of these methods, such as accuracy, convergence, and computational cost [11-13]. Specially, there is a defect that both these methods require precise probability distributions of the random variables based on a great amount of experimental samples as in many engineering applications and the samples are often limited. It causes the distributions of some variables not being precisely known. Generally, we can only obtain the variation ranges of some parameters based on the limited data and descript the stochastic characteristic of them with subjective assumptions [16]. It is likely to bring about a serious error in structural reliability analysis [17-20].

In real-world engineering, it is obvious that random and interval variables coexist frequently [21, 22]. Quantifying both types of the abovementioned uncertainty is called hybrid reliability analysis (HRA) [1]. HRA has been an important issue in the research field in structural reliability 
[22]. In recent years, many research studies have been carried out on the HRA methods for structure [23-26]. The following numerical methods have been proposed: the function approximation technique [27], the probabilistic transformation technique [28-30], the iterative rescaling method [31], the probability bounds approach [32], the mixed perturbation Monte Carlo (MC) method [33], the optimization algorithm with single-layer nesting [34, 35], two-layer nesting [36, 37], and the complex nesting [22], among others [38-40]. Nevertheless, the HRA with FORM is still in its infancy, as there are some calculating problems in the practical applications. Algorithms with high efficiency and robust convergence must be developed [21, 22].

Hence, a new procedure, based on first-order reliability method (FORM), is proposed to deal with random and interval hybrid variables. This procedure can significantly improve the computational efficiency for the abovementioned HRA problem, preserving sufficient precision. In the proposed method, we involve the distance coordinate and the cosine of the angle coordinate in a polar space. In this space, the hybrid problem is reduced to standard reliability problem, where an $n$-dimensional limit-state function is defined only in terms of the two random variables. And the probability density functions (PDFs) of the two variables can be obtained by the PDFs of random variables and boundaries of interval variables. Finally, the interval of failure probability is efficiently calculated with the integral method, for the new bidimensional function.

The main contributions of this paper lie twofold in the following:

(1) In the framework of the proposed method, the novel procedure is more useful and efficient in analyzing the hybrid reliability problem with randomness and interval.

(2) The new PDFs of two nonlinear features have been deduced, using the probability distribution of randomness and the range of intervals. It is distinct from the assumption of uniform density function model for interval variables. The new PDFs of the two features contribute to the evaluation of the lower and upper probability of failure, respectively.

The remainder of this paper is organized as follows. A brief summary of the classical structural reliability analysis with FORM is presented in Section 2. The whole efficient reliability analysis method has been proposed in Section 3. In Section 3.1, the polar transformation with random and interval uncertainty has been discussed, and the distribution of two new variables in the polar space has been derived. The new calculated models and algorithm procedures for the range of failure probability have been proposed in Section 3.2. Finally, two numerical applications to demonstrate its efficiency and practicability are presented. Firstly, the hybrid reliability problem with one simple linear explicit expression is used to prove that this method is effective and accurate, due to low analytical complexity of the linear limit-state function. Secondarily, the method is applied to deal with the hybrid reliability problem in a real structural system, in this specific case a spacecraft docking lock, to demonstrate the effectiveness of the proposed method.

\section{Traditional Structural Reliability Analysis}

In the reliability design and analysis of structures, the limit-state function $G(\mathbf{X})$ is always constructed according to specified requirements. Here, the $n$-dimensional variable vector $\mathbf{X}=\left(x_{1}, x_{2}, \ldots, x_{n}\right)^{T}$ denotes the independent random variables that affect the function, such as load, material properties, geometry, dimensions, and environmental factors. $G(\mathbf{X})$ can be used to determine if the structural system works normally by checking whether $G(\mathbf{X})>0$ or not. The probability of occurrence of failure event $P_{\mathrm{f}}$ is obtained as follows [2, 3]:

$$
P_{\mathrm{f}}=\operatorname{Pr}\{G(\mathbf{X}) \leq 0\},
$$

where $\operatorname{Pr}\{\bullet\}$ is the probabilistic measure. According to the dual event $G(\mathbf{X})>0$, the probability of reliability $P_{\text {reliability }}$ can be formulated as follows:

$$
P_{\text {reliability }}=1-\operatorname{Pr}(G(\mathbf{X}) \leq 0)=\operatorname{Pr}(G(\mathbf{X})>0),
$$

which can be computed by the following integral:

$$
\operatorname{Pr}(G(\mathbf{X})>0)=\int_{G(\mathbf{X})>0} f_{\mathbf{X}}(\mathbf{X}) d \mathbf{X},
$$

where $f_{\mathbf{X}}(\mathbf{X})$ denotes the joint PDF of the random variables $\mathbf{X}$. Generally, the above integral cannot be solved analytically because of its complex integration boundary and the high dimensions of $\mathbf{X}$. Therefore, some approximation techniques have been well established to compute the integral efficiently, which include the well-known first-order reliability method (FORM) [16]. In FORM, by a following transformation from the original space of $\mathbf{X}$ to an independent standard normal space of $\mathbf{u}$, the limit-state function becomes as follows:

$$
G(\mathbf{X})=G(T(\mathbf{u}))
$$

where $T$ is the transformation that converts $\mathbf{u}$ into $\mathbf{X}$. Additionally, this equation will in the sequel be represented simply as

$$
g(\mathbf{u})=G(\mathbf{X})=G(T(\mathbf{u}))
$$

with the understanding that there is an underlying transformation to the Gaussian space $\mathbf{u}$ from the given space $\mathbf{X}$.

Thus, the integration in (3) can be rewritten as

$$
\operatorname{Pr}(g(\mathbf{u})>0)=\int_{g(\mathbf{u})>0} f_{\mathbf{u}}(\mathbf{u}) d \mathbf{u} .
$$

$f_{\mathbf{u}}(\mathbf{u})$ is the $n$-dimensional standard Gaussian density function. By using the reliability index approach, the above equation can be further changed to the following optimization problem [23]:

$$
\begin{array}{lr}
\min & \beta=\|\mathbf{u}\|, \\
\text { s.t. } & g(\mathbf{u})=0,
\end{array}
$$


where $\|\bullet\|$ denotes the norm of a vector. The optimum $\mathbf{u}^{*}$ is called the design point where the joint PDF reaches a maximum value among all of the points on the limit-state function. $\beta=\left\|\mathbf{u}^{*}\right\|$ is a reliability index which geometrically represents a minimum distance from the origin to the limit-state surface in $\mathbf{u}$ space.

Despite that this problem and its solution are well known, for the ensuing developments, it is necessary to recall its main steps. The first-order Taylor expansion of the limit-state function about a general point $\mathbf{u}^{+}$can be written as follows:

$$
\widehat{g}(\mathbf{u}) \approx g\left(\mathbf{u}^{+}\right)+\nabla g\left(\mathbf{u}^{+}\right)\left(\mathbf{u}-\mathbf{u}^{+}\right),
$$

where $\nabla g\left(\mathbf{u}^{+}\right)$stands for $\nabla g(\mathbf{u})$ evaluated at $\mathbf{u}^{+}$. In FORM, the design point is the solution of the optimization problem (7). Its value is as follows:

$$
\mathbf{u}^{*}=\beta \boldsymbol{\alpha},
$$

where $\boldsymbol{\alpha}=\nabla g\left(\mathbf{u}^{*}\right) /\left\|\nabla g\left(\mathbf{u}^{*}\right)\right\|$ is the design point unit vector, which is normal to the tangent hyper plane at the design point.

Moreover, we can suggest that a source of difficulties for solving the structural reliability problem by any method is its dimensionality, determined by the number of random variables $n$ [41]. In essence, this is due to the fact that the estimation of the normally very small probability failure requires a large number of samples in high dimensional spaces, either for establishing a surrogate of the limit-state function or for MC solution. This also makes the visualization of the reliability problem impossible, which is a desirable goal for the design practice [29]. Therefore, some methods are proposed for reducing the dimensionality of the problem to only two dimensions, by means of a polar representation for random samples used in MC simulation, based on the vector of the center of mass of the failure probability and the design point [23]. There are two nonlinear features: (a) their distance to the origin and (b) the cosine of the angle they make with the design point unit vector $\boldsymbol{\alpha}$. Moreover, these methods are developed to estimate the reliability of a structure system in which the input variables are modeled using any representation provided by possibility distributions, intervals, probability boxes, CDFs, or Dempster-Shafer structures based on random set theory [29]. The two new variables are given as follows:

$$
\begin{aligned}
& v_{1}=r=\|\mathbf{u}\|_{2}, \\
& v_{2}=\cos \theta=\frac{(\mathbf{u}, \boldsymbol{\alpha})}{\|\mathbf{u}\|\|\boldsymbol{\alpha}\|}=\frac{(\mathbf{u}, \boldsymbol{\alpha})}{\|\mathbf{u}\|}=\frac{(\mathbf{u}, \boldsymbol{\alpha})}{v_{1}}, \\
& \boldsymbol{\alpha}=\frac{\nabla g\left(\mathbf{u}^{*}\right)}{\left\|\nabla g\left(\mathbf{u}^{*}\right)\right\|} .
\end{aligned}
$$

For the large number $n, v_{1}$ obeys a chi distribution [41]:

$$
\phi_{1}\left(v_{1} ; n\right)=\frac{2^{1-n / 2} v_{1}^{(n-1)}}{\Gamma n / 2} \exp \left(-\frac{v_{1}^{2}}{2}\right), \quad v_{1}>0
$$

and $v_{2}$ obeys the following distribution:

$$
\begin{array}{r}
\phi_{2}\left(v_{2}\right)=\frac{\left(\sin ^{n-2}\left(\arccos v_{2}\right)+\sin ^{n-2}\left(\pi-\arccos v_{2}\right)\right)}{\sqrt{1-v_{2}^{2}} \int_{0}^{\pi} \sin ^{n-2} \boldsymbol{\alpha} d \boldsymbol{\alpha}}, \\
-1<v_{2}<1, n \geq 2 .
\end{array}
$$

It was derived by Hurtado that the two new variables are independent $[23,41]$. In order to avoid the serious error of the reliability analysis brought by subjective assumptions on description of the uncertainty characteristics, such as the uniform distribution or truncated Gaussian distribution representation for interval uncertainty, the new polar transformation with random and interval uncertainty is presented in next the section.

\section{Efficient Reliability Analysis Method with FORM}

3.1. Polar Transformation with Random and Interval Uncertainty. In structural reliability problem, let us assume that the limit-state function is $g(\mathbf{x}, \mathbf{y})=g\left(x_{1}, x_{2}, \ldots, x_{n}, y_{1}\right.$, $\left.y_{2}, \ldots, y_{m}\right)$, where $\mathbf{x}=\left(x_{1}, x_{2}, \ldots, x_{n}\right)$ is a $n$-dimensional vector of random variables and $\mathbf{y}=\left(y_{1}, y_{2}, \ldots, y_{m}\right)$ is a $m$-dimensional vector of interval variables. In terms of the standard independent Gaussian vector $\mathbf{u}=\left(u_{1}, u_{2}, \ldots, u_{n}\right)$ and the normalized independent interval vector $\boldsymbol{\delta}=\left(\delta_{1}, \delta_{2}, \ldots, \delta_{m}\right)$ $\in[-1,1]$, the limit-state function becomes as follows:

$$
g(\mathbf{x}, \mathbf{y})=g(T(\mathbf{u}), \tilde{T}(\boldsymbol{\delta})),
$$

where $T$ is the standard Gaussian transformation that converts $\mathbf{u}$ into $\mathbf{x}$ and $\tilde{T}$ is the normalized interval transformation that converts $\delta$ into $\mathbf{y}$. In order to keep the notation uncluttered, this equation will in the sequel be represented simply as

$$
g(\mathbf{x}, \mathbf{y})=g(\boldsymbol{\omega}) .
$$

With $\boldsymbol{\omega}=(\mathbf{u}, \boldsymbol{\delta})$, the structural reliability problem is defined in the $n+m$ dimensional space $\mathbf{w}$ of $n$ independent standard Gaussian variables and $m$ normalized interval variables. The main purpose of this method is to transform it to a problem of only two dimensions. By (10),, and, we can use the polar transformation from space $\omega$ to the polar space by the two nonlinear features:

$$
\begin{aligned}
& \widehat{v}_{1}=r=\|\boldsymbol{\omega}\|_{2}=\sqrt{\sum_{i=1}^{n} u_{i}^{2}+\sum_{j=1}^{m} \delta_{i}^{2}}, \\
& \widehat{v}_{2}=\cos \theta=\frac{(\boldsymbol{\omega}, \widehat{\boldsymbol{\alpha}})}{\|\boldsymbol{\omega}\|\|\widehat{\boldsymbol{\alpha}}\|}=\frac{(\boldsymbol{\omega}, \widehat{\boldsymbol{\alpha}})}{\|\boldsymbol{\omega}\|}=\frac{(\boldsymbol{\omega}, \widehat{\boldsymbol{\alpha}})}{v_{1}}, \\
& \widehat{\boldsymbol{\alpha}}=\frac{\nabla g\left(\boldsymbol{\omega}^{*}\right)}{\left\|\nabla g\left(\boldsymbol{\omega}^{*}\right)\right\|},
\end{aligned}
$$

where $\omega^{*}$ is the solution of the new optimization problem

$$
\begin{array}{ll}
\min & \beta=\|\boldsymbol{\omega}\|, \\
\text { s.t. } & g(\boldsymbol{\omega})=0, \\
& \delta \in[-1,1] .
\end{array}
$$


Hence, in the polar space, the distribution of two new variables can be derived as follows.

For $\forall n, m \in N$ ( $N$ is the nonnegative integer domain), $n$ is the number of the random variables and $m$ is the number of the interval variables.

(1) Like $v_{2}$ in Section 2, $\widehat{v}_{2}$ obeys a distribution:

$$
\begin{array}{r}
\phi_{2}(y)=\frac{\left(\sin ^{n+m-2}(\arccos y)+\sin ^{n+m-2}(\pi-\arccos y)\right)}{\sqrt{1-y^{2}} \int_{0}^{x} \sin ^{n+m-2} \alpha d \alpha}, \\
-1 \leq y \leq 1 .
\end{array}
$$

(2) For $\widehat{v}_{1}=\sqrt{\sum_{i=1}^{n} u_{i}^{2}+\sum_{j=1}^{m} \delta_{i}^{2}}, \quad \widehat{v}_{1} \in(0,+\infty)$, let $\Delta=$ $\sum_{j=1}^{m} \delta_{i}^{2}$, with $\forall \delta_{i} \in[-1,1](i=1,2, \ldots, m)$; it is derived that $0 \leq \sum_{j=1}^{m} \delta_{i}^{2} \leq m$. As $u_{i}(i=1,2, \ldots, n)$ is the independent standard Gaussian variable, $\eta_{1}=$ $v_{1}^{2}-\Delta=\sum_{i=1}^{n} u_{i}^{2}, \Delta \in[0, m]$, obeys the $\chi^{2}(n)$ distribution shown as follows:

$$
f\left(\eta_{1}\right)=\frac{2^{1-n / 2} \eta_{1}^{(n-1)}}{\Gamma n / 2} \exp \left(-\frac{\eta_{1}^{2}}{2}\right) \quad\left(\eta_{1}>0\right),
$$

where $n$ is the number of the random variables. Hence, for $\forall \Delta \in[0, m], \widehat{v}_{1}$ obeys the distribution written as follows:

$$
\begin{aligned}
\phi_{1}(x, \Delta)=\frac{2^{1-n / 2}\left(x^{2}-\Delta\right)^{(n-1) / 2}}{\Gamma n / 2} & \exp \left(-\frac{x^{2}-\Delta}{2}\right), \\
x & \geq \sqrt{\Delta}, \Delta \in[0, m],
\end{aligned}
$$

where $m$ is the number of the interval variables. And the cumulative distribution function (CDF) of $\widehat{v}_{1}$ with $\Delta$ denotes $F_{v_{1}}(x, \Delta)$. It can be obtained that $F_{v_{1}}$ $(x, \Delta)$ is nonincreasing for the variable $\Delta$.

Proof. Let $\tau=x^{2}-\Delta, \tau \in[0, \infty]$, the partial derivative of $F_{v_{1}}$ $(x, \Delta)$ for $\Delta$ can be written as follows:

$$
\begin{aligned}
\frac{\partial F_{v_{1}}(x, \Delta)}{\partial \Delta} & =\frac{\partial F_{v_{1}}(\tau)}{\partial \Delta}=\frac{\partial F_{v_{1}}(\tau)}{\partial \tau} \frac{\partial \tau}{\partial \Delta} \\
& =\frac{\tau^{(n-1) / 2}}{2^{n / 2} \Gamma n / 2} \exp \left(-\frac{\tau}{2}\right) \frac{1}{\sqrt{\tau+\Delta}} .
\end{aligned}
$$

Considering $\forall \Delta,\left(\tau^{(n-1) / 2} / 2^{n / 2} \Gamma(n / 2)\right) \geq 0, \exp (-(\tau / 2))>$ 0 , and $(1 / \sqrt{\tau+\Delta})>0$, thus it is obvious that $\left(\partial F_{v_{1}}(x, \Delta) /\right.$ $\partial \Delta) \leq 0$. Hence, $F_{v_{1}}(x, \Delta)$ is nonincreasing for the variable $\Delta$.

Moreover, because of the two new variables $\widehat{v}_{1}, \widehat{v}_{2}$ independent in the polar space, with the limit-state function $G\left(\widehat{v}_{1}, \widehat{v}_{2}\right)$, the probability of failure $P_{\text {failure }}$ in the structure can be written as follows:

$$
\begin{array}{r}
P_{\text {failure }}(\Delta)=\int_{-1}^{1} \int_{\sqrt{\Delta}=\left\{\hat{v} \mid G\left(\widehat{v}_{1}, \hat{v}_{2}\right) \leq 0\right\}}^{+\infty} \phi_{1}\left(\widehat{v}_{1}, \Delta\right) \phi_{2}\left(\widehat{v}_{2}\right) d \widehat{v}_{1} d \widehat{v}_{2}, \\
\Delta \in[0, m],
\end{array}
$$

where $\phi_{1}$ obeys (19) and $\phi_{2}$ obeys (17).

Considering the nonincreasing of CDF of $v_{1}$ for $\Delta$, the interval of $P_{\mathrm{f}}$ is $\left[\underline{P}_{\mathrm{f}}, \bar{P}_{\mathrm{f}}\right]$; the lower boundary $\underline{P}_{\mathrm{f}}(\Delta=m)$ and the upper boundary $\bar{P}_{\mathrm{f}}(\Delta=0)$ can be calculated by

$$
\begin{aligned}
& \bar{P}_{\mathrm{f}}=\int_{\Omega_{\nu}=\left\{\hat{v} \mid G\left(\widehat{v}_{1}, \hat{v}_{2}\right) \leq 0\right\}}^{1} \int_{1}^{+\infty} \phi_{1}\left(\widehat{v}_{1}, \Delta\right) \phi_{2}\left(\widehat{v}_{2}\right) d \widehat{v}_{1} d \widehat{v}_{2} \quad(\Delta=0), \\
& \underline{P}_{\mathrm{f}}=\int_{\substack{-1 \\
\Omega_{v}=\left\{\hat{v} \mid G\left(\widehat{v}_{1}, \hat{v}_{2}\right) \leq 0\right\}}}^{+\infty} \phi_{1}\left(\widehat{v}_{1}, \Delta\right) \phi_{2}\left(\widehat{v}_{2}\right) d \widehat{v}_{1} d \widehat{v}_{2} \quad(\Delta=m),
\end{aligned}
$$

where $\phi_{1}$ obeys (20) and $\phi_{2}$ obeys (18).

3.2. The Efficient Method with FORM. Let us consider the first-order approximation $\hat{g}(\omega)$ at the design point $\omega^{*}$ of the limit-state function $g(\boldsymbol{\omega})$ given by (8) with the last term disregarded, where $\omega^{*}=\left(u^{*}{ }_{1}, u^{*}{ }_{2}, \ldots, u^{*}{ }_{n}, \delta_{1}{ }^{*}, \delta_{2}{ }^{*}, \ldots\right.$, $\left.\delta_{m}{ }^{*}\right)$ obtained by the optimization problem (16). $\hat{g}(\boldsymbol{\omega})$ can be written as follows:

$$
\widehat{g}(\boldsymbol{\omega}) \approx g\left(\boldsymbol{\omega}^{*}\right)+\left.\sum_{i=1}^{n} \frac{\partial g}{\partial u_{i}}\right|_{\boldsymbol{\omega}^{*}}\left(u_{i}-u_{i}^{*}\right)+\left.\sum_{j=1}^{m} \frac{\partial g}{\partial \delta_{j}}\right|_{\boldsymbol{\omega}^{*}}\left(\delta_{j}-\delta_{j}^{*}\right) .
$$

Let

$$
\begin{aligned}
d & =\frac{g\left(\omega^{*}\right)-\left.\sum_{i=1}^{n}\left(\partial g / \partial u_{i}\right)\right|_{\omega^{+}} u_{i}^{*}-\left.\sum_{j=1}^{m}\left(\partial g / \partial \delta_{j}\right)\right|_{\omega^{\omega}} \delta_{j}^{*}}{\left[\sum_{i=1}^{n}\left(\left.\left(\partial g / \partial u_{i}\right)\right|_{\omega^{*}}\right)^{2}+\sum_{j=1}^{m}\left(\left.\left(\partial g / \partial \delta_{j}\right)\right|_{\omega^{*}}\right)^{2}\right]^{1 / 2}} \\
\widehat{v}_{1} & =\sqrt{\sum_{i=1}^{n} u_{i}^{2}+\sum_{j=1}^{m} \delta_{i}^{2}} \\
\widehat{v}_{2} & =\cos \angle\left(\boldsymbol{\omega}^{+}, \boldsymbol{\omega}\right) \\
& =\frac{\left.\sum_{k=1}^{n+m}\left(\partial g / \partial \omega_{i}\right)\right|_{\omega^{*}} \omega_{i}}{\widehat{v}_{1} \sqrt{\sum_{k=1}^{n+m}\left(\left.\left(\partial g / \partial \omega_{k}\right)\right|_{\omega^{*}}\right)^{2}}} \\
& =\frac{\left.\sum_{i=1}^{n}\left(\partial g / \partial u_{i}\right)\right|_{\omega^{*}} u_{i}+\left.\sum_{j=1}^{m}\left(\partial g / \partial \delta_{j}\right)\right|_{\omega^{*}} \delta_{j}}{\widehat{v}_{1} \sqrt{\sum_{i=1}^{n}\left(\left.\left(\partial g / \partial u_{i}\right)\right|_{\omega^{*}}\right)^{2}+\sum_{j=1}^{m}\left(\left.\left(\partial g / \partial \delta_{j}\right)\right|_{\omega^{*}}\right)^{2}}}
\end{aligned}
$$

where

$$
\boldsymbol{\omega}^{+}=\frac{\boldsymbol{\omega}^{*}}{\left\|\boldsymbol{\omega}^{*}\right\|}=\frac{\left(\left.\left(\partial g / \partial \omega_{1}\right)\right|_{\boldsymbol{\omega}^{*}}, \ldots,\left.\left(\partial g / \partial \omega_{n+m}\right)\right|_{\omega^{*}}\right)}{\sqrt{\sum_{k=1}^{n+m}\left(\left.\left(\partial g / \partial \omega_{k}\right)\right|_{\omega^{*}}\right)^{2}}} .
$$


Equation (26) can be written as follows by taking (24):

$$
\begin{aligned}
\widehat{g}(\boldsymbol{\omega}) & \approx g\left(\boldsymbol{\omega}^{*}\right)+\left.\sum_{i=1}^{n} \frac{\partial g}{\partial u_{i}}\right|_{\boldsymbol{\omega}^{*}}\left(u_{i}-u_{i}^{*}\right)+\left.\sum_{j=1}^{m} \frac{\partial g}{\partial \delta_{j}}\right|_{\boldsymbol{\omega}^{*}}\left(\delta_{j}-\delta_{j}^{*}\right) \\
& =d * D+D * v_{1} v_{2}=G\left(v_{1}, v_{2}\right)
\end{aligned}
$$

where

$$
\begin{aligned}
& D=\left[\sum_{i=1}^{n}\left(\left.\frac{\partial g}{\partial u_{i}}\right|_{\omega^{*}}\right)^{2}+\sum_{j=1}^{m}\left(\left.\frac{\partial g}{\partial \delta_{j}}\right|_{\omega^{*}}\right)^{2}\right]^{1 / 2}, \\
& d=\frac{g\left(\boldsymbol{\omega}^{*}\right)-\left.\sum_{i=1}^{n}\left(\partial g / \partial u_{i}\right)\right|_{\omega^{*}} u_{i}^{*}-\left.\sum_{j=1}^{m}\left(\partial g / \partial \delta_{j}\right)\right|_{\omega^{*}} \delta_{j}^{*}}{D} .
\end{aligned}
$$

Distinctly, $D=\left[\sum_{i=1}^{n}\left(\left.\left(\partial g / \partial u_{i}\right)\right|_{\omega^{*}}\right)^{2}+\sum_{j=1}^{m}\left(\left.\left(\partial g / \partial \delta_{j}\right)\right|_{\omega^{*}}\right)^{2}\right]^{1 / 2}$ $\neq 0$; in the polar space, the new safe domain becomes $d+v_{1} v_{2}>0$, the failure domain becomes $d-v_{1} v_{2} \leq 0$, and the limit-state surface is $d-v_{1} v_{2}=0$.

We can calculate the interval of the failure probability $\left[\underline{P}_{f}, \bar{P}_{\mathrm{f}}\right]$ in the structure with (29) and (31) as follows.

(1) The lower boundary of failure probability $\underline{P}_{\mathrm{f}}$ :

$$
\underline{P}_{\mathrm{f}}=\int_{\Omega=\left\{d-v_{1} v_{2} \leq 0\right\}}^{1} \int_{\sqrt{m}}^{\infty} \phi_{2}\left(v_{2}\right) \phi_{1}\left(v_{1}, m\right) d v_{1} d v_{2} \text {. }
$$

(2) The upper boundary of failure probability $\bar{P}_{f}$ :

$$
\bar{P}_{\mathrm{f}}=\int_{\substack{\Omega \\-1}}^{1} \int_{0}^{\infty} \phi_{2}\left(v_{2}\right) \phi_{1}\left(v_{1}, 0\right) d v_{1} d v_{2},
$$

where $\phi_{1}$ obeys (19) and $\phi_{2}$ obeys (17).

The method proceeds as follows:

(1) For the random variables $x_{1}, x_{2}, \ldots, x_{n}$ and interval variables $y_{1}, y_{2}, \ldots, y_{m}\left(y_{i} \in\left[y_{i}^{L}, y_{i}^{U}\right], \quad i=1,2, \ldots, m\right)$ in the limit-state function $g(\mathbf{x}, \mathbf{y})$ of a structure, convert $\mathbf{x}=\left(x_{1}, x_{2}, \ldots, x_{n}\right)$ into the standard independent Gaussian vector $\mathbf{u}=\left(u_{1}, u_{2}, \ldots, u_{n}\right)$ and $\mathbf{y}=\left(y_{1}, y_{2}, \ldots, y_{m}\right)$ into the normalized independent interval vector $\boldsymbol{\delta}=\left(\delta_{1}, \delta_{2}, \ldots, \delta_{m}\right) \in[-1,1]$. The limit-state function $g(\mathbf{x}, \mathbf{y})$ becomes $g(\boldsymbol{\omega})$ with $\omega=(\mathbf{u}, \delta)$.

(2) Let the initial design point be $\omega^{*(0)}=\left(u_{1}^{*(0)}, u_{2}^{*(0)}, \ldots\right.$, $\left.u_{n}^{*(0)}, \delta_{1}^{*(0)}, \ldots, \delta_{m}^{*(0)}\right)$, obtained by the optimization problem (16).

(3) Calculate the gradient $\partial g /\left.\partial \omega_{i}\right|_{\omega^{*(0)}}$ of the function $g(\boldsymbol{\omega})$ at the design point $\boldsymbol{\omega}^{*(0)}$.

(4) Transform the function $g(\boldsymbol{\omega})$ into $G^{(0)}\left(v_{1}, v_{2}\right)=$ $d^{(0)} \times D^{(0)}+D^{(0)} \times v_{1} v_{2}$ in the polar space and calculate the parameters

$$
\begin{aligned}
D^{(0)} & =\left[\sum_{i=1}^{n}\left(\left.\frac{\partial g}{\partial u_{i}}\right|_{\omega^{*(0)}}\right)^{2}+\sum_{j=1}^{m}\left(\left.\frac{\partial g}{\partial \delta_{j}}\right|_{\omega^{*(0)}}\right)^{2}\right]^{1 / 2}, \\
d^{*(0)} & =\frac{g\left(\omega^{*(0)}\right)+\left.\sum_{i=1}^{n}\left(\partial g / \partial u_{i}\right)\right|_{\omega^{*}} u_{i}^{*(0)}+\left.\sum_{j=1}^{m}\left(\partial g / \partial \delta_{j}\right)\right|_{\omega^{*}} \delta_{j}^{*(0)}}{D^{(0)}} .
\end{aligned}
$$

(5) In the polar space, use (32) and (33) to calculate the initial interval of failure probability $\left[\underline{P}_{\mathrm{f}}^{(0)}, \bar{P}_{\mathrm{f}}^{(0)}\right]$ of the structure written as follows:

$$
\begin{gathered}
\underline{P}_{\mathrm{f}}^{(0)}=\int_{\substack{-1 \\
\left\{d^{(0)}-v_{1} v_{2} \leq 0\right\}}}^{1} \int_{\sqrt{m}}^{+\infty} \phi_{2}\left(v_{2}\right) \phi_{1}\left(v_{1}, m\right) d v_{1} d v_{2}, \\
\bar{P}_{\mathrm{f}}^{(0)}=\int_{\Omega_{v}=\left\{\mathbf{v} \mid d^{(0)}-v_{1} v_{2} \leq 0\right.}^{1} \int_{0}^{+\infty} \phi_{2}\left(v_{2}\right) \phi_{1}\left(v_{1}, 0\right) d v_{1} d v_{2} .
\end{gathered}
$$

(6) Calculate the new gradient $\omega_{k}^{+(l)}(k=1,2, \ldots, n+m)$ $(l=1,2, \ldots$,$) by the following:$

$$
\omega_{k}^{+(l)}=\frac{\left.\left(-\partial g / \partial \omega_{k}\right)\right|_{\omega_{k}^{*}(l-1)}}{\left[\sum_{k=1}^{n+m}\left(\left.\left(\partial y / \partial \omega_{k}\right)\right|_{\omega_{k}^{*(l-1)}}\right)^{2}\right]^{1 / 2}} \quad(l=1,2, \ldots,) .
$$

(7) Calculate the new design point $\omega^{*(l)}=\left[\omega_{1}^{*(l)}, \omega_{2}^{*(l)}, \ldots\right.$, $\left.\omega_{n+m}^{*(l)}\right]$ with $\omega_{k}^{+(l)}(k=1,2, \ldots, n+m)(l=1,2, \ldots$,$) by$ the following:

$$
\begin{aligned}
\omega_{k}^{*(l)}= & \boldsymbol{\omega}^{*(l-1)}+\left\|\boldsymbol{\omega}^{*(l-1)}\right\| \alpha_{k}^{(l)}=\boldsymbol{\omega}^{*(l-1)} \\
& +\left\|\boldsymbol{\omega}^{*(l-1)}\right\| \frac{-\left.\left(\partial g / \partial \omega_{k}\right)\right|_{\boldsymbol{\omega}_{k}^{*(l-1)}}}{\left[\sum_{k=1}^{n+m}\left(\left.\left(\partial y / \partial \omega_{k}\right)\right|_{\boldsymbol{\omega}_{k}^{*}(l-1)}\right)^{2}\right]^{1 / 2}} .
\end{aligned}
$$


In the polar space, the parameters $d^{(l)}$ and $D^{(l)}$ by (35) and (36), respectively, are written as follows:

$$
\begin{aligned}
d^{(l)} & =\frac{g\left(\omega^{*(l)}\right)+\left.\sum_{k=1}^{n+m}\left(\partial g / \partial \omega_{k}\right)\right|_{\omega_{k}^{*(l)}} \omega_{k}^{*(l)}}{\left[\sum_{k=1}^{n+m}\left(\left.\left(\partial g / \partial \omega_{k}\right)\right|_{\omega_{k}^{*(l)}}\right)^{2}\right]^{1 / 2}}, \\
D^{(l)} & =\left[\sum_{k=1}^{n+m}\left(\left.\frac{\partial g}{\partial \omega_{k}}\right|_{\omega^{*(l)}}\right)^{2}\right]^{1 / 2} .
\end{aligned}
$$

And the new limit-state function is $G^{(l)}\left(v_{1}, v_{2}\right)=d^{(l)} *$ $D^{(l)}-D^{(l)} * v_{1} v_{2}$.

(8) Calculate the interval of failure probability $\left[\underline{P}_{\mathrm{f}}^{(l)}, \bar{P}_{\mathrm{f}}^{(l)}\right]$ of the structure by the following:

$$
\begin{aligned}
& \underline{P}_{\mathrm{f}}^{(l)}=\int_{-1}^{1} \int_{\sqrt{m}}^{+\infty} \phi_{1}\left(v_{1}, m\right) \phi_{2}\left(v_{2}\right) d v_{1} d v_{2}, \\
& \Omega_{v}=\left\{\mathbf{v} \mid d^{(l)}-v_{1} v_{2} \leq 0\right\} \\
& \bar{P}_{\mathrm{f}}^{(l)}=\int_{\substack{\Omega_{v}=\left\{\mathbf{v} \mid d^{(l)}-v_{1} v_{2} \leq 0 \\
1\right.}}^{+\infty} \phi_{1}\left(v_{1}, 0\right) \phi_{2}\left(v_{2}\right) d v_{1} d v_{2} \text {. }
\end{aligned}
$$

(9) For the robust convergence of this procedure, let the iterative error of the calculation be $\varepsilon$. If $\left|\bar{P}_{\mathrm{f}}^{(l)}-\bar{P}_{\mathrm{f}}^{(l-1)}\right| /$ $\left(1-\bar{P}_{\mathrm{f}}^{(1)}\right) \leq \varepsilon$ and $\left|\underline{P}_{\mathrm{f}}^{(l)}-\underline{P}_{\mathrm{f}}^{(l-1)}\right| /\left(1-\underline{P}_{\mathrm{f}}^{(1)}\right) \leq \varepsilon$, stop calculating.

Otherwise, go to step 2. Set the new design point $\boldsymbol{\omega}^{*(l)}=\left[\omega_{1}^{*(l)}, \omega_{2}^{*(l)}, \cdots, \omega_{n+m}^{*(l)}\right]$ to be the initial design point.

\section{Numerical Examples}

4.1. Numerical Example with Linear Limit-State Function. (1) In the structural reliability problems, let the limit-state function of a structure be $G(\mathbf{u}, \boldsymbol{\delta})=1-(1 / n+m)\left(\sum_{i=1}^{n} u_{i}+\right.$ $\left.\sum_{j=1}^{m} \delta_{j}\right)$, with the random variable $u_{i}(i=1,2, \ldots, n)$ obeying the standard normal distribution $N(0,1)$ and the interval variable $\delta_{j}(j=1,2, \ldots, m)$ in the range of $[0,1]$. They are independent of each other.

With the polar transformation, let

$$
\begin{aligned}
& v_{2}=\frac{\sqrt{1 /(n+m)}\left(\sum_{i=1}^{n} u_{i}+\sum_{j=1}^{m} \delta_{j}\right)}{\sqrt{\sum_{i=1}^{n} u_{i}^{2}+\sum_{j=1}^{m} \delta_{j}^{2}}}, \\
& v_{1}=\sqrt{\sum_{i=1}^{n} u_{i}^{2}+\sum_{j=1}^{m} \delta_{j}^{2}} .
\end{aligned}
$$

And $G(\mathbf{u}, \boldsymbol{\delta})$ can be transformed to $G(\mathbf{v})=1-$

\begin{tabular}{|c|c|c|c|}
\hline \multirow{2}{*}{ Methods } & & \multicolumn{2}{|c|}{ Failure probability } \\
\hline & & $\begin{array}{l}\text { Upper } \\
\text { bound }\end{array}$ & $\begin{array}{l}\text { Lower } \\
\text { bound }\end{array}$ \\
\hline \multirow{8}{*}{$\begin{array}{l}\text { MC } 10^{8} \\
\text { (confidence 95\%) }\end{array}$} & $n=1, m=1$ & 0.0932 & 0.0785 \\
\hline & $n=5, m=5$ & $5.110 e-04$ & $3.498 e-06$ \\
\hline & $n=10, m=5$ & $3.512 e-05$ & $3.550 e-06$ \\
\hline & $n=5, m=10$ & $3.376 e-05$ & $3.332 e-16$ \\
\hline & $n=5, m=20$ & $3.153 e-07$ & $3.599 e-20$ \\
\hline & $n=10, m=15$ & $3.201 e-07$ & $2.684 e-22$ \\
\hline & $n=15, m=10$ & $3.096 e-07$ & $2.910 e-16$ \\
\hline & $n=20, m=5$ & $3.141 e-07$ & $2.469 e-06$ \\
\hline \multirow{8}{*}{ The proposed method } & $n=1, m=1$ & 0.1012 & 0.0694 \\
\hline & $n=5, m=5$ & $4.906 e-04$ & $3.699 e-06$ \\
\hline & $n=10, m=5$ & $3.452 e-05$ & $3.538 e-06$ \\
\hline & $n=5, m=10$ & $3.452 e-05$ & $3.326 e-16$ \\
\hline & $n=5, m=20$ & $3.100 e-07$ & $3.669 e-20$ \\
\hline & $n=10, m=15$ & $3.100 e-07$ & $2.699 e-22$ \\
\hline & $n=15, m=10$ & $3.100 e-07$ & $2.901 e-16$ \\
\hline & $n=20, m=5$ & $3.100 e-07$ & $2.368 e-06$ \\
\hline
\end{tabular}
$\sqrt{(2 /(n+m))} v_{1} v_{2}$ in polar space, where the PDF of the random variable $v_{1}$ is (18), and the PDF of $v_{2}$ is (20).

We can see that $n+m$ uncertain variables are transformed to two uncertain variables in the polar space.
TABLE 1: Bounds of the failure probability estimated by two methods.

TABLE 2: The relative error and iteration number of the proposed method.

\begin{tabular}{lccc}
\hline \multirow{2}{*}{ Situation } & \multicolumn{2}{c}{ Relative error } & \multirow{2}{*}{ Iteration number } \\
& Upper bound & Lower bound & \\
\hline$n=1, m=1$ & $8.58 \%$ & $11.6 \%$ & 20 \\
$n=5, m=5$ & $3.99 \%$ & $5.73 \%$ & 68 \\
$n=10, m=5$ & $1.73 \%$ & $0.34 \%$ & 111 \\
$n=5, m=10$ & $2.25 \%$ & $0.16 \%$ & 124 \\
$n=5, m=20$ & $1.66 \%$ & $1.96 \%$ & 191 \\
$n=10, m=15$ & $3.16 \%$ & $0.54 \%$ & 182 \\
$n=15, m=10$ & $0.13 \%$ & $0.29 \%$ & 163 \\
$n=20, m=5$ & $1.31 \%$ & $4.07 \%$ & 171 \\
\hline
\end{tabular}

Subsequently, we will discuss the calculated interval of failure probability with different $n$ and $m$ as shown in the following tables. For comparison purposes, a full-interval MC simulation involving the generation of 10,000 random realizations of input parameters $\mathbf{u}$ and the computation of the failure probability for each of them with 10,000 samples of vector $\boldsymbol{\delta}$ was performed.

The relevant information about the two methods applied is summarized in Tables 1 and 2. Table 1 indicates that the estimates calculated by the proposed method in the paper are exactly in the interval Monte Carlo sense. Also, by the relative errors of the bounds in Table 2, it can be seen that the method succeeds in giving accurate reliability analyses with the maximum value $11.6 \%$. This example shows the efficiency of the method, inasmuch as only 191 


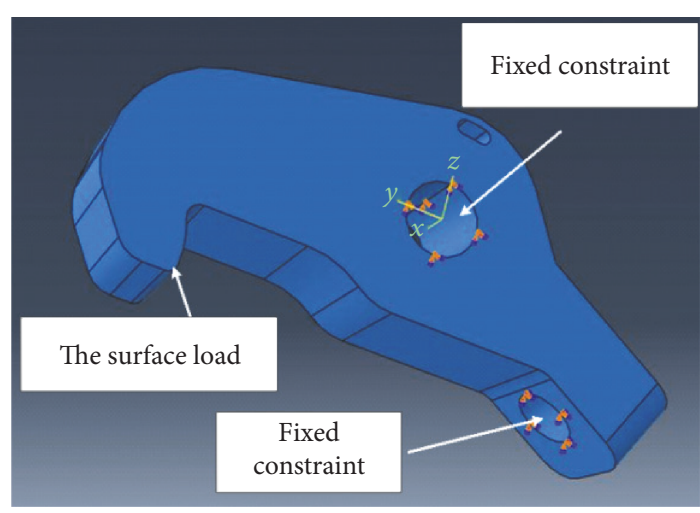

FIGURE 1: FEA model of structural latch.

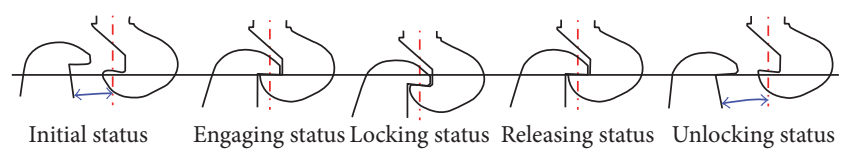

FIgURE 2: The workflow of the structural latch.

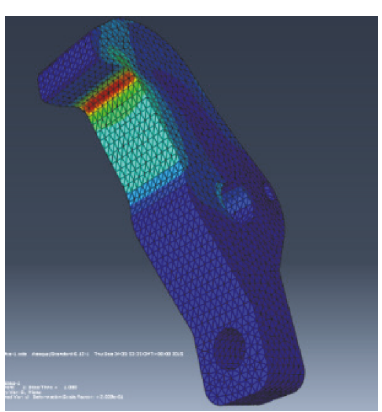

(a)

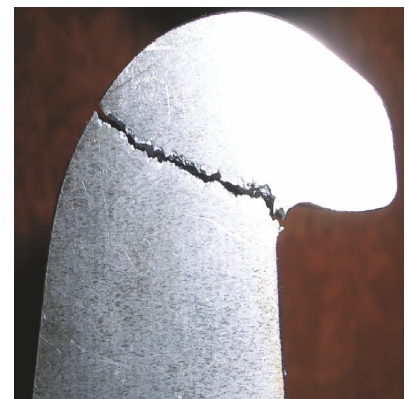

(b)
FIGURE 3: The stress nephogram of the structural latch (a) and the contrast strength test (b).

iterations (maximum value) were required in comparison to the 100,000,000 sample evaluations required by interval MC in the evaluation of $\underline{P}_{\mathrm{f}}$ and $\bar{P}_{\mathrm{f}}$, achieving the same precision of the latter (interval MC).

On the other hand, from the same total number of uncertain variables with different $n$ random variables and $m$ interval variables, it is suggested that the number of interval variables influences the results stronger than randomness. From the different total numbers of uncertain variables, it is suggested that the number influences the efficiency (iteration).

4.2. Structural Latch of Space Docking Mechanism. Consider the structural latch of space docking mechanism shown in Figure 1. It is an important part of the docking mechanism, which is the key structure to realize the rigid connection and separation between two space vehicles. The connection force is generated by the interaction between the series of latches in two different docking mechanisms.
The working procedure of the workflow of structural latch is shown in Figure 2.

During the procedure of locking every structural latch, the load $F$ is an interval variable with the range of $[30,40]$ $\mathrm{kN}$. The modulus of elasticity of the material $E$ is a normal variable with the mean $117,000 \mathrm{MPa}$ and standard deviation $1170 \mathrm{MPa}$, and the cross section depth $d$ is a normal variable with the mean $5 \mathrm{~mm}$ and standard deviation $0.05 \mathrm{~mm}$. Also, there are two random variables Poisson ratio $\mu \sim N(0.3,0.03)$ and the material density $\rho \sim N(4.81 \mathrm{~g} / \mathrm{c}$ $\left.\mathrm{m}^{3}, 0.481 \mathrm{~g} / \mathrm{cm}^{3}\right)$. The reliability problem is defined by the limit-state function

$$
g=\sigma_{\max }-\sigma(F, E, \mu, \rho, d)
$$

where $\sigma_{\max }$ is the yield strength with the constant and $\sigma$ $(F, E, \mu, \rho, d)$ is the contact stress on the connected surface of the two latches.

It is supposed that the four random variables are independent. A simplified finite element analysis (FEA) model is created to compute the contact stress $\sigma$ (Figure 3), with 25,642 elements.

The proposed algorithm in this paper was employed to perform the new FORM analysis. After 127 iterations, the lower and upper bound of the failure probability were calculated as $[4.3580 e-06,4.3930 e-05]$. It coincides with the result calculated using Monte Carlo simulation, inasmuch as all of the failure samples of the simulation were correctly identified. However, the relative errors are $8.12 \%$ and $11.4 \%$, which indicate that this method employs the linear Taylor series to preserve the efficiency, sacrificing the accuracy requirements. The calculated result for the structural latch with comparison is summarized in Table 3.

The difference between the results of MC and the proposed method is caused by the nonlinearity of the limitstate function. However, the error of the upper bound is only $8.12 \%$ and that of the lower bound is $11.4 \%$, which indicates that the nonlinearity of this problem is not too strong, but still directly influences the result precision. Compared with the relative error, the results of the presented method can be accepted. On the other hand, the evaluation number of FEA for the proposed method is only 223. Since each evaluation of the FEA model takes almost $10 \mathrm{~min}$, the increased computational cost for MC would be more than 15 days. Therefore, if we pay more attention to the computational cost in this problem, then this proposed method in this paper is preferred, although losing better accuracy.

\section{Conclusion}

In this paper, an efficient method for the hybrid reliability analysis of structures for random and interval uncertainty is presented. It has been shown that the design point concept of probability-based reliability analysis can still be applied in this article. Specifically, the unit vector of the point is the direction of the rapid changes of the output variable implied in the limit-state function. In order to avail of this property and reduce the computation cost, a bidimensional mapping of two nonlinear features is established in the polar space: 
TABLE 3: The calculated result for the structural latch with comparison.

\begin{tabular}{|c|c|c|c|c|c|}
\hline \multirow{2}{*}{ Method } & \multicolumn{2}{|c|}{ Failure probability } & \multicolumn{2}{|c|}{ Relative error } & \multirow{2}{*}{ Iteration number } \\
\hline & Upper bound & lower bound & Upper bound & lower bound & \\
\hline MC $10^{8}$ (confidence 95\%) & $4.7801 e-05$ & $3.9120 e-06$ & l & l & l \\
\hline The proposed method & $4.3930 e-05$ & $4.3580 e-06$ & $8.12 \%$ & $11.4 \%$ & 127 \\
\hline
\end{tabular}

(a) the distance to the origin and (b) the cosine of the angle with the design point unit vector. In this case, the hybrid problem is reduced to standard reliability problem, where $n$-dimensional limit-state function with the linear Taylor series is approximated around the design point defined only in terms of two random variables, obeying new PDFs with the number of random and interval variables. They are distinct from the assumption of uniform density function model for interval variables and contribute to the evaluation of the lower and upper probability of failure, respectively. The numerical experiments confirm the solid theoretical foundation, the agreeable accuracy, and the low computational cost of the proposed methodology.

\section{Conflicts of Interest}

The authors declare that they have no competing interests.

\section{Acknowledgments}

This study was supported by the National Basic Research Program of China (no. 2013CB733002) and the National Natural Science Foundation of China (no. 51675026).

\section{References}

[1] L. Wang, X. J. Wang, and Y. Xia, "Hybrid reliability analysis of structures with multi-source uncertainties," Acta Mechanica, vol. 225, no. 2, pp. 413-430, 2014.

[2] R. Melchers, Structural Reliability Analysis and Prediction, John Wiley, New York, NY, USA, 1999.

[3] I. Elishakoff, Probabilistic Theory of Structures, Florida Atlantic University, Dover, NY, USA, 1999.

[4] G. C. Marano and G. Quaranta, "A new possibilistic reliability index definition," Acta Mechanica, vol. 210, no. 3-4, pp. 291303, 2010 .

[5] B. K. Low, "FORM, SORM, and spatial modeling in geotechnical engineering," Structural Safety, vol. 49, pp. 56-64, 2014.

[6] Z. Nizamani, V. J. Kurian, and M. S. Liew, "Determination of environmental load factors for ISO 19902 code in offshore Malaysia using FORM structural reliability method," Ocean Engineering, vol. 92, pp. 31-43, 2014.

[7] R. H. Lopez, L. F. F. Miguel, I. M. Belo, and J. E. Souza Cursi, "Advantages of employing a full characterization method over FORM in the reliability analysis of laminated composite plates," Composite Structures, vol. 107, no. 1, pp. 635-642, 2014.

[8] C. Jiang, S. Han, M. Ji, and X. Han, "A new method to solve the structural reliability index based on homotopy analysis," Acta Mechanica, vol. 226, no. 4, pp. 1067-1083, 2015.
[9] E. G. Abdelouafi, K. Benaissa, and K. Abdellatif, "Reliability analysis of reinforced concrete buildings: comparison between FORM and ISM," Procedia Engineering, vol. 114, pp. 650-657, 2015.

[10] Z. Zhang, C. Jiang, G. G. Wang, and X. Han, "First and second order approximate reliability analysis methods using evidence theory," Reliability Engineering \& System Safety, vol. 137, pp. 40-49, 2015.

[11] K. Breitung, "40 years FORM: some new aspects?," Probabilistic Engineering Mechanics, vol. 42, pp. 71-77, 2015.

[12] R. Rackwitz, "Reliability analysis: a review and some perspectives," Structural Safety, vol. 23, no. 4, pp. 365-395, 2001.

[13] R. H. Lopez, A. J. Torii, L. F. F. Miguel, and J. E. Souza Cursi, "Overcoming the drawbacks of the FORM using a full characterization method," Structural Safety, vol. 54, pp. 57-63, 2015.

[14] A. D. Kiureghian, "The geometry of random vibrations and solutions by FORM and SORM," Probabilistic Engineering Mechanics, vol. 15, no. 1, pp. 81-90, 2000.

[15] M. Barbato, G. Qu, and J. P. Conte, "A new multidimensional visualization technique for limit-state surfaces in nonlinear finite-element reliability analysis," Journal of Engineering Mechanics, vol. 136, no. 11, pp. 1390-1400, 2010.

[16] C. Jiang and G. Y. LuX. Han and L. X. Liu, "A new reliability analysis method for uncertain structures with random and interval variables," International Journal of Mechanics and Materials in Design, vol. 8, no. 2, pp. 169-182, 2012.

[17] I. Elishakoff and M. Ohsaki, Optimization and AntiOptimization of Structures under Uncertainty, Imperial College Press, London, 2010.

[18] X. J. Wang, L. Wang, I. Elishakoff, and Z. Qiu, "Probability and convexity concepts are not antagonistic," Acta Mechanica, vol. 219, no. 1-2, pp. 45-64, 2011.

[19] L. Wangi, X. Wang, and Y. Xia, "Hybrid reliability analysis of structures with multi-source uncertainties," Acta Mechanica, vol. 225, no. 2, pp. 413-430, 2014.

[20] W. Yao, X. Chen, Y. Huang, Z. Gurdal, and M. van Tooren, "Sequential optimization and mixed uncertainty analysis method for reliability-based optimization," AIAA Journal, vol. 51, no. 51, pp. 2266-2277, 2013.

[21] S. J. Xie, B. S. Pan, and X. P. Du, "An efficient hybrid reliability analysis method with random and interval variables," Engineering Optimization, vol. 48, no. 9, pp. 1459-1473, 2016.

[22] U. Alibrandi and C. G. Koh, "Stochastic dynamic analysis of floating production systems using the first order reliability method and the secant hyperplane method," Ocean Engineering, vol. 137, pp. 68-67, 2017.

[23] J. E. Hurtado and D. A. Alvarez, "The encounter of interval and probabilistic approaches to structural reliability at the design point," Computer Methods in Applied Mechanics and Engineering, vol. 225-228, pp. 74-94, 2012.

[24] K. Zaman, S. Rangavajhala, M. P. Mcdonald, and S. Mahadevan, "A probabilistic approach for representation 
of interval uncertainty," Reliability Engineering \& System Safety, vol. 96, no. 1, pp. 117-130, 2011.

[25] X. P. Du, "Unified uncertainty analysis by the first order reliability method," Journal of Mechanical Design, vol. 130, no. 9, article 091401, 2008.

[26] S. X. Guo and Z. Z. Lu, "Hybrid probabilistic and nonprobabilistic model of structural reliability," Journal of Mechanical Strength, vol. 24, no. 4, pp. 524-526, 2002.

[27] R. C. Penmetsa and R. V. Grandhi, "Efficient estimation of structural reliability for problems with uncertain intervals," Computers \& Structures, vol. 80, no. 12, pp. 1103-1112, 2002.

[28] S. Sankararaman and S. Mahadevan, "Separating the contributions of variability and parameter uncertainty in probability distributions," Reliability Engineering \& System Safety, vol. 112, no. 112, pp. 187-199, 2013.

[29] D. A. AlvarezJ. E. Hurtado, "An efficient method for the estimation of structural reliability intervals with random sets, dependence modeling and uncertain inputs," Computers \& Structures, vol. 142, pp. 54-63, 2014.

[30] J. E. Hurtado, "Assessment of reliability intervals under input distributions with uncertain parameters," Probabilistic Engineering Mechanics, vol. 32, no. 4, pp. 80-92, 2013.

[31] J. W. Hall and J. Lawry, "Generation, combination and extension of random set approximations to coherent lower and upper probabilities," Reliability Engineering \& System Safety, vol. 85, no. 1, pp. 89-101, 2004.

[32] D. R. Karanki, H. S. Kushwaha, A. K. Verma, and S. Ajit, "Uncertainty analysis based on probability bounds (p-box) approach in probabilistic safety assessment," Risk Analysis, vol. 29, no. 5, pp. 662-675, 2009.

[33] W. Gao, D. Wu, C. M. Song, F. Tin-Loi, and X. Li, "Hybrid probabilistic interval analysis of bar structures with uncertainty using a mixed perturbation Monte-Carlo method," Finite Elements in Analysis and Design, vol. 47, no. 7, pp. 643-652, 2011.

[34] S. J. Xie, B. S. Pan, and X. P. Du, "A single-loop optimization method for reliability analysis with second order uncertainty," Engineering Optimization, vol. 47, no. 8, pp. 1125-1139, 2014.

[35] D. Yoo and I. Lee, "Sampling-based approach for design optimization in the presence of interval variables," Structural and Multidisciplinary Optimization, vol. 49, no. 2, pp. 253266, 2014.

[36] J. Guo and X. P. Du, "Reliability sensitivity analysis with random and interval variables," International Journal for Numerical Methods in Engineering, vol. 78, no. 13, pp. 15851617, 2010.

[37] Y. J. Luo, Z. Kang, and A. Li, "Structural reliability assessment based on probability and convex set mixed model," Computers \& Structures, vol. 87, no. 21-22, pp. 1408-1415, 2009.

[38] X. P. Du, A. Sudjianto, and B. Q. Huang, "Reliability-based design under the mixture of random and interval variables," Journal of Mechanical Design, vol. 127, no. 6, pp. 1068-1076, 2003.

[39] Z. P. Qiu and J. Wang, "The interval estimation of reliability for probabilistic and non-probabilistic hybrid structural system," Engineering Failure Analysis, vol. 17, no. 5, pp. 1142$1154,2010$.
[40] R. Chowdhury and B. N. Rao, "Hybrid high dimensional model representation for reliability analysis," Computer Methods in Applied Mechanics and Engineering, vol. 198, no. 5-8, pp. 753-765, 2009.

[41] J. E. Hurtado, "Dimensionality reduction and visualization of structural reliability problems using polar features," Probabilistic Engineering Mechanics, vol. 29, no. 7, pp. 16-31, 2012. 


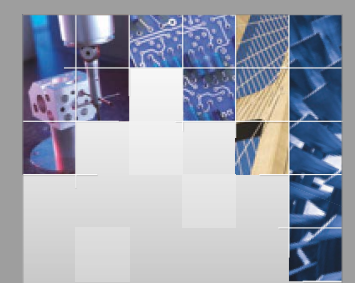

\section{Enfincering}
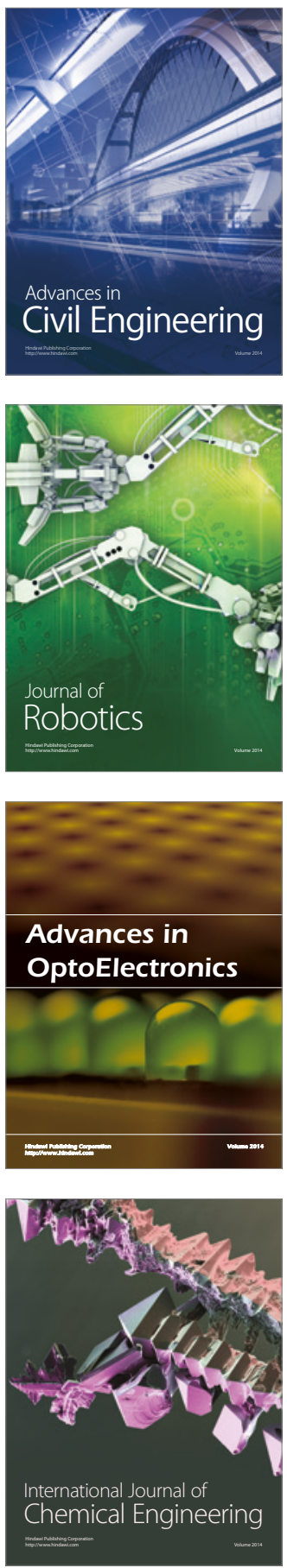

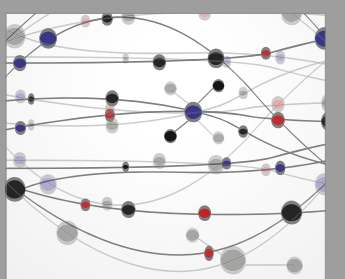

The Scientific World Journal

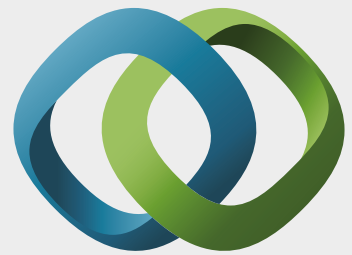

\section{Hindawi}

Submit your manuscripts at

https://www.hindawi.com
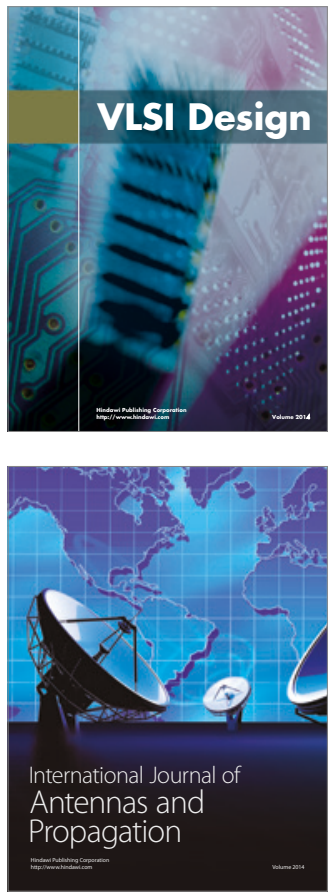

\section{Rotating}

Machinery
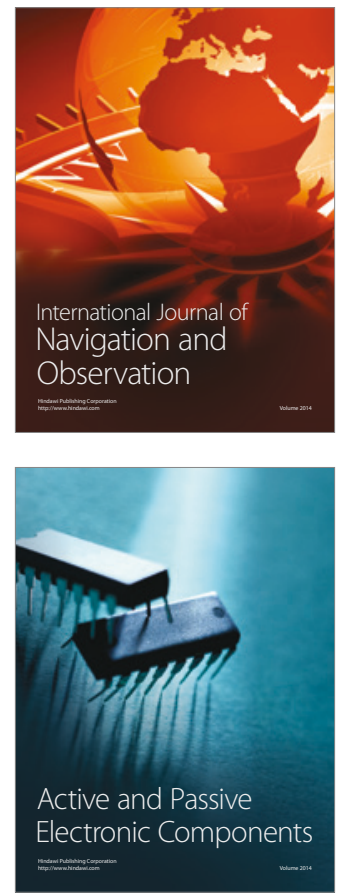
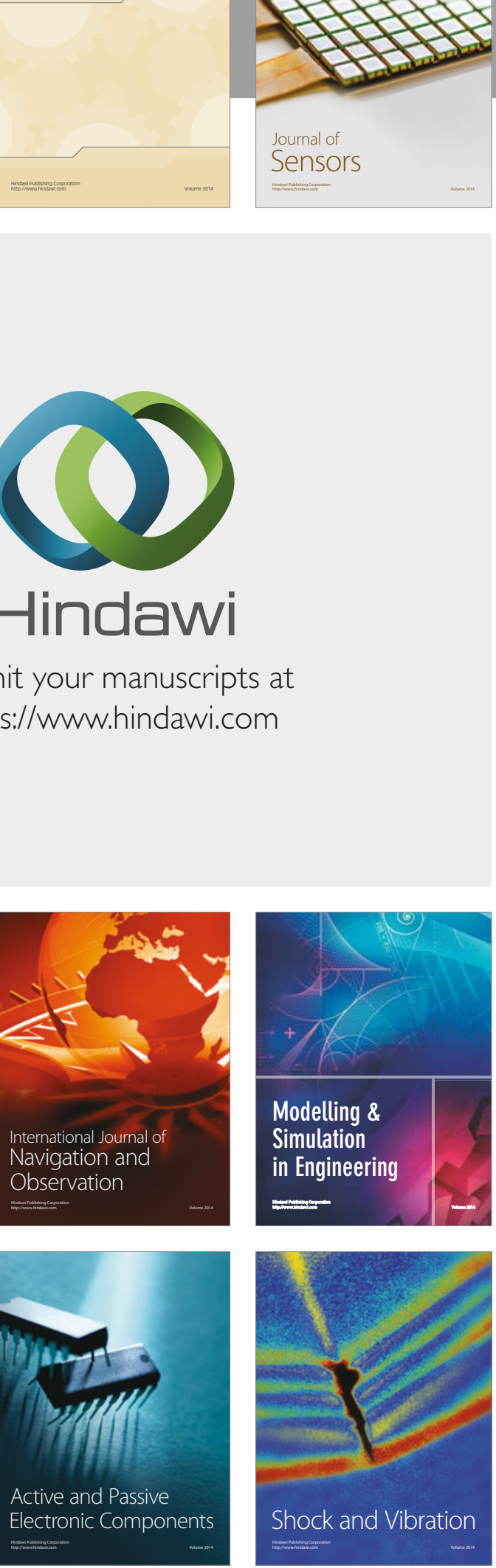
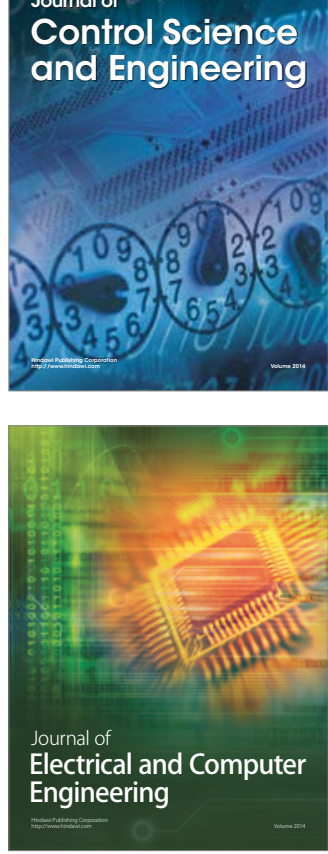

Distributed

Journal of

Control Science

and Engineering
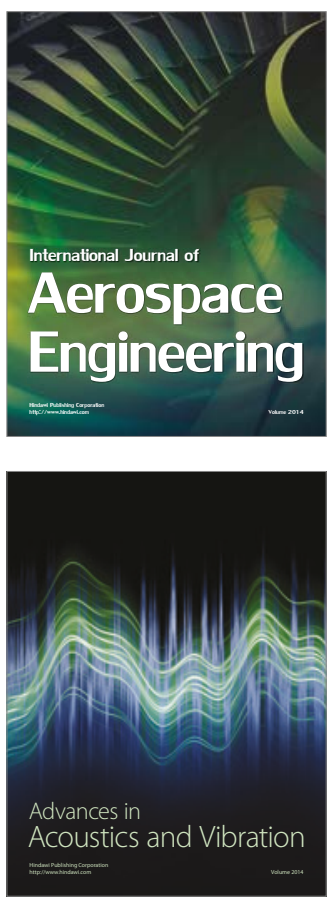

Sensor Networks 